\title{
A Domain Decomposition Multilevel Preconditioner for Interpolation with Radial Basis Functions
}

\author{
Gundolf Haase $^{1}$, Dirk Martin ${ }^{2}$, Patrick Schiffmann ${ }^{2}$, and Günter Offner ${ }^{2}$ \\ 1 Institute for Mathematics and Scientific Computing, University of Graz, Austria \\ gundolf .haase@uni-graz.at, \\ WWW home page: http://imsc.uni-graz.at/haasegu \\ 2 AVL List GmbH, Graz, Austria
}

\begin{abstract}
We present the reasonableness of the extension of a two-level domain decomposition method to a multilevel method as a preconditioner for interpolation with radial basis functions (RBF) on distributed memory systems. The arising subproblems are efficiently solved using the FGP algorithm, a method that is well-suited for shared memory settings.
\end{abstract}

\section{Introduction}

Many scientific and industrial simulation problems involve interpolation over numerous data sites as a subtask. Interpolation with radial basis functions (RBF) is an interpolation method applicable with (almost) no conditions on the distribution of the data sites and in arbitrary dimensions, that features favourable smoothness and convergence properties. Nevertheless, a straightforward application is hindered by the ill-conditioning of the resulting dense systems of linear equations arising from the interpolation task $[2,3,15]$.

We use RBF interpolation for the deformation of computational domains in $\mathbb{R}^{3}$ as proposed in [4]. The originating simulation tasks require the utilisation of distributed memory systems, thus a distributed solution for the interpolation task is demanded. We pursue our approach in [12] by extending the presented two-level method to a multilevel method. As the initial approach, our method is based on $[2,13]$. The arising subproblems are efficiently solved by a preconditioned Krylov subspace projection method suitable for shared memory settings [8,9], the FGP algorithm.

The remaining paper is organized as follows. Section 2 gives an introduction to RBF interpolation. Section 3 features preconditioning methods for shared memory and distributed memory settings. This includes the extension of a twolevel method to a multilevel method. We present numerical results in section 4 and annotate some conclusions in section 5 . 


\section{Interpolation with Radial Basis Functions}

For detailed discussions of the analysis on RBF interpolation we refer to the books $[6,18]$. A comprehensive view over aspects of theory and application is given in [10].

The general setting for RBF interpolation is a given set of data sites $X=\left\{x_{i}\right\}_{i=1}^{N}$ from a domain $\Omega \subseteq \mathbb{R}^{d}$ and a set of associated real function values $f_{i}=f\left(x_{i}\right)$. Sought is an approximating function $s: \Omega \rightarrow \mathbb{R}$ by interpolation $\left.s\right|_{X}=\left.f\right|_{X}$ of the form

$$
s(x)=\sum_{i=1}^{N} \lambda_{i} \phi\left(\left\|x-x_{i}\right\|\right)+p(x),
$$

with real coefficients $\lambda_{i}$, a radial function $\Phi$, and a polynomial term $p \in \mathbb{P}_{d}^{k-1}$. In general, a function $\Phi: \mathbb{R}^{d} \rightarrow \mathbb{R}$ is called radial if there exists a univariate function $\phi:[0, \infty) \rightarrow \mathbb{R}$ such that $\Phi(\boldsymbol{x})=\phi\left(\|\boldsymbol{x}\|_{2}\right)$. The polynomial term $p$ is required for the existence and uniqueness of a solution, the required degree depends on the choice of the basis function $\phi$. In case the choice of the basis function requires a polynomial term, the given set of points $X$ has to be unisolvent with respect to polynomials of the corresponding degree. A set $X \subset \mathbb{R}^{d}$ is called unisolvent for $\mathbb{P}_{d}^{k-1}$, if $\left.p\right|_{X}=0 \Rightarrow p \equiv 0$ for polynomials $p \in \mathbb{P}_{d}^{k-1}$.

Let $p_{j}, j=1, \ldots, M$ be a basis of $\mathbb{P}_{d}^{k-1}$. The interpolant $s$ can be determined by inserting the given data sites and function values in (1). Demanding a side condition for the coefficients of the required polynomial term leads to a dense system of linear equations:

$$
\begin{array}{cl}
\sum_{i=1}^{N} \lambda_{i} \phi\left(\left\|x_{i}-x_{k}\right\|\right)+\sum_{j=1}^{M} \pi_{j} p_{j}\left(x_{k}\right)=f\left(x_{k}\right), & 1 \leq k \leq N \\
\sum_{i=1}^{N} \lambda_{i} p_{l}\left(x_{i}\right)=0, & 1 \leq l \leq M
\end{array}
$$

or, in short notation

$$
A \boldsymbol{x}=\boldsymbol{b}:=\left(\begin{array}{cc}
\Phi & \Pi \\
\Pi^{\top} & 0
\end{array}\right)\left(\begin{array}{l}
\boldsymbol{\lambda} \\
\boldsymbol{\pi}
\end{array}\right)=\left(\begin{array}{l}
\boldsymbol{f} \\
\mathbf{0}
\end{array}\right) .
$$

We restrict our numerical examples to the choice of the multiquadric basis function

$$
\phi(x)=\sqrt{x^{2}+c^{2}}
$$

with a real scaling parameter $c$ to allow comparability with earlier results $[12,14]$. The multiquadric basis function requires a constant polynomial term.

\section{Preconditioning RBF Interpolation}

The system (4) is w.l.o.g dense and ill-conditioned. Therefore, the numerical solution requires iterative methods with a suitable preconditioning, if the system size surpasses certain limits. 


\subsection{The FGP Algorithm}

The FGP algorithm $[8,9,11]$ is a preconditioned Krylov subspace projection method based on the semi-inner product

$$
\langle s, t\rangle_{\phi}=\varrho \boldsymbol{\lambda}^{\top} \Phi \boldsymbol{\mu}
$$

with $s(x)=\sum_{i=1}^{N} \lambda_{i} \phi\left(\left\|x-x_{i}\right\|\right)+\sum_{j=1}^{M} \alpha_{j} p_{j}(x)$ for $\lambda_{i}, \alpha_{j} \in \mathbb{R}$ and $t(x)=$ $\sum_{i=1}^{N} \mu_{i} \phi\left(\left\|x-x_{i}\right\|\right)+\sum_{j=1}^{M} \beta_{j} p_{j}(x)$ for $\mu_{i}, \beta_{j} \in \mathbb{R}$, induced by the radial basis function $\phi$. $\Phi$ denotes the associated kernel to $\phi$. The factor $\varrho \in\{-1,1\}$ depends on the choice of the basis function.

The included preconditioning is based on an approximation of the Lagrange basis of the spanned function space, which can be obtained by solving the interpolation tasks

$$
\hat{u}_{k}\left(x_{i}\right)=\sum_{\ell=1}^{N} \zeta_{k, \ell} \phi\left(\left\|x_{i}-x_{\ell}\right\|\right)+\sum_{j=1}^{M} \pi_{k, j} p_{j}\left(x_{i}\right):=\delta_{i k}, \quad \text { for } i, k=1, \ldots, N,
$$

where $\delta_{i k}$ denotes the Kronecker-delta. As an approximation, the functions $\hat{u}_{k}$, $k=1, \ldots, N$ are computed on subsets of $X$, that contain not more than $q$ interpolation centers. Generally the relation $q \ll N$ holds. These subsets are called Lagrange-sets $(\mathcal{L}$-sets). The $\mathcal{L}$-sets are chosen such that they span subsets of $X$ in various scales.

The FGP algorithm is well-suited for shared memory settings. We applied this method effectively on CPUs and accellerators $[12,14]$. Nevertheless, the distribution of the $\mathcal{L}$-sets impedes the application for distributed memory settings.

\subsection{Domain Decompostion Methods}

Beatson et al. present a list of four building blocks that they consider the 'essential ingredients for a domain decomposition interpolatory fitter' [2]:

1. A space subdivision method.

2. A solution method for small interpolation subproblems.

3. A fast evaluation of the RBF interpolations occuring at various spaces.

4. An outer iteration.

For the construction of a domain decomposition method (DDM), the given set of interpolation centers $X$ is subdivided into the overlapping subdomains $X_{j}, j=1, \ldots, D$ such that $X=\bigcup_{j=1}^{D} X_{j}$ and $X_{i} \cap X_{j} \neq \emptyset$. Associated with this subdivision is a set of non-overlapping subdomains $\tilde{X}_{j}, j=1, \ldots, D$ such that $X=\bigcup_{j=1}^{D} \tilde{X}_{j}$, where $\tilde{X}_{j} \subset X_{j}$ and $\tilde{X}_{i} \cap \tilde{X}_{j}=\emptyset$. The cardinalities of the subsets are denoted by $N_{j}=\left|X_{j}\right|$ and $\tilde{N}_{j}=\left|\tilde{X}_{j}\right|$ respectively.

Let $\Re_{s} \in \mathbb{R}^{N \times N_{s}}$ denote the restriction matrix projecting a vector $\boldsymbol{x}$ from a domain $X$ onto a vector $\boldsymbol{x}_{s}=\Re_{s} \boldsymbol{x}$ on the subdomain $X_{s}$ and $\tilde{\Re}_{s} \in \mathbb{R}^{N \times \tilde{N}_{s}}$ 
denote the restriction matrix which restricts a vector $\boldsymbol{x}$ from domain $X$ onto a vector $\tilde{\boldsymbol{x}}_{s}=\tilde{\Re}_{s} x$ on the subdomain $\tilde{X}_{s}$.

For the non-overlapping subdivision a vector over the domain $X$ can be composed by applying the transposed mapping operations $\tilde{\Re}_{s}^{\top}$ on the local vectors $\boldsymbol{x}_{s}$

$$
\boldsymbol{x}=\sum_{s=1}^{D} \tilde{\Re}_{s}^{\top} \boldsymbol{x}_{s}
$$

Beatson et. al. present a two-level method [2] that also employs a coarse grid correction. The selection of the set of the coarse grid interpolation centers $X_{C}$ is denoted as $\Re_{C} \in \mathbb{R}^{N \times N_{C}}$, where $N_{C}:=\left|X_{C}\right|$. The coarse grid nodes are chosen such that $X_{C} \cap X_{s} \neq \emptyset$ for $s=1, \ldots, D$.

Retaining the notation from (4), we write $A_{s}, \boldsymbol{x}_{s}, \boldsymbol{b}_{s}$ for the systems over subdomains. The restriction matrices $\Re_{s}$ (and $\tilde{\Re}_{s}$ ) are extended by zero blocks to the matrices $R_{s}$ (and $\tilde{R}_{s}$ )

$$
R_{s}:=\left(\begin{array}{cc}
\Re r_{s} & 0 \\
0 & 0
\end{array}\right) \in \mathbb{R}^{(N+M) \times\left(N_{s}+M\right)} \text { and } \tilde{R}_{s}:=\left(\begin{array}{cc}
\tilde{\Re}_{s} & 0 \\
0 & 0
\end{array}\right) \in \mathbb{R}^{(N+M) \times\left(\tilde{N}_{s}+M\right)} .
$$

We denote the projection of a vector in $\mathbb{R}^{N}$ onto $\left(\mathbb{P}_{d}^{k-1}\right)^{\perp}$ as $\mathfrak{P} \in \mathbb{R}^{N \times N}$. As for the restriction matrices we extend the projection in order to spare the polynomial term to an operator

$$
P:=\left(\begin{array}{ll}
\mathfrak{P} & 0 \\
0 & 0
\end{array}\right) \in \mathbb{R}^{(N+M) \times(N+M)} .
$$

A notable difference to the extended restriction matrices defined above is the matrix $\hat{R}$, since we need to carry the update for the polynomial part from the coarse grid solution:

$$
R_{C}:=\left(\begin{array}{cc}
\mathfrak{R}_{C} & 0 \\
0 & 0
\end{array}\right) \in \mathbb{R}^{(N+M) \times\left(N_{C}+M\right)} \text { and } \hat{R}_{C}:=\left(\begin{array}{cc}
\mathfrak{R}_{C} & 0 \\
0 & I
\end{array}\right) \in \mathbb{R}^{(N+M) \times\left(N_{C}+M\right)} \text {. }
$$

We can now write the iteration step as follows:

$$
\begin{gathered}
\boldsymbol{x}^{(i+1 / 2)}=\boldsymbol{x}^{(i)}+P \sum_{j=1}^{D} \tilde{R}_{j}^{\top} A_{j}^{-1} R_{j}\left(\boldsymbol{f}-A \boldsymbol{x}^{(i)}\right) \\
\boldsymbol{x}^{(i+1)}=\boldsymbol{x}^{(i+1 / 2)}+\hat{R}_{C}^{\top} A_{C}^{-1} R_{C}\left(\boldsymbol{f}-A \boldsymbol{x}^{(i+1 / 2)}\right) .
\end{gathered}
$$

The presented algorithm can be regarded as a two-level Schwarz method that is multiplicative between the levels and a restricted additive Schwarz method (RASM) within the fine level [16]. A notable difference to general Schwarz methods is the required correction of the fine-level coefficients that assures (3).

It is common for applications of Schwarz methods to replace the correction over the subdomains with approximate solvers [16, sec. 1.2]. Ling and Kansa 
proposed to use approximate solvers for a DDM for radial basis function interpolation [13].

The theory of preconditioned Krylov subspace projection methods requires the preconditioning operator to be constant for all iterations. In general, this does not hold if the approximated solution is achieved by an iterative method itself. As shown in section 4, our numerical tests show only a slight increase of the required iteration numbers, backing up the statements about the applicability of approximated solutions over subdomains in $[13,16]$.

In [12], we implemented the two-level block Jacobi approach using the data division that was predetermined by our application, in particular finite volume discretizations with one cell-layer overlap. For the generation of the coarse representation we chose a coarsening factor of $1 / 8$. We approximately solve the arising subproblems applying the FGP algorithm. Both, the outer iteration of the DDM and the inner iteration of the FGP algoritm require the computation of a matrix-vector product with a dense system matrix. We approximate this matrix-vector product with a multipole method $[1,7]$.

In order to extend this approach to a general multilevel method we define subsets $X_{l}, l=1, \ldots, L$ of the given set $X$, such that $X_{l} \subset X_{l+1}$ for $l=1, \ldots, L-1$ and $X_{L} \equiv X$. Each level $l>1$ is subdivided into overlapping subdomains $X_{j, l}, j=1, \ldots, D_{l}$ and the associated non-overlapping subdivision $\tilde{X}_{j, l}, j=1, \ldots, D_{l}$. The notation of the cardinalities is similar to the notation above $N_{j, l}=\left|X_{j, l}\right|$ and $\tilde{N}_{j, l}=\left|\tilde{X}_{j, l}\right|$. The notation of the restriction matrices is also extended by the current level in a similar manner.

For the RASM within the levels $l>1$, we again require a projection of the composed coefficient vector onto the subspace $\left(\mathbb{P}_{d}^{k-1}\right)^{\perp}$.

The extension to a general multilevel method on $L$ levels now has the form

$$
\begin{aligned}
\boldsymbol{x}^{(i+1 / L)} & =\boldsymbol{x}^{(i)}+P \sum_{j=1}^{D_{L}} \tilde{R}_{j, L}^{\top} A_{j, L}^{-1} R_{j, L}\left(\boldsymbol{f}-A \boldsymbol{x}^{(i)}\right) \\
\boldsymbol{x}^{(i+2 / L)} & =\boldsymbol{x}^{(i+1 / L)}+P \sum_{j=1}^{D_{L-1}} \tilde{R}_{j, L-1}^{\top} A_{j, L-1}^{-1} R_{j, L-1}\left(\boldsymbol{f}-A \boldsymbol{x}^{(i+1 / L)}\right) \\
\vdots & \\
\boldsymbol{x}^{(i+1)} & =\boldsymbol{x}^{(i+(L-1) / L)}+\hat{R}_{1}^{\top} A_{1}^{-1} R_{1}\left(\boldsymbol{f}-A \boldsymbol{x}^{(i+(L-1) / L)}\right) .
\end{aligned}
$$

For the extension of our implementation, the predetermined data division was replaced with an octree as hierarchical data structure in $\mathbb{R}^{3}$. Each nonempty box represents a subdomain, the overlap is constructed from contributions of the neighbor boxes. Each refinement level of the octree represents a level of the multilevel method. As for the two-level implementation we employ the FGP algorithm as local solution method. The computation of the involved matrixvector products is approximated with a multipole method in a straightforward manner. 


\section{Numerical Examples}

In this section we present iteration counts for non-preconditioned Krylov subspace projection methods compared to our implementation of the FGP algorithm and the presented multilevel method and timing measurements for our distributed multilevel implementation. We imposed test function F3 [5] as boundary conditions on $N$ nodes distributed over a sphere.

Table 1 compares the iteration counts for the CG method, the GMRES method, the FGP algorithm with $\mid \mathcal{L}$-set $\mid=50$, and variations of our multilevel approach for solving the system to a $L_{2}$-error less than $10^{-5}$. The value of the overlap is given relatively to the box size of the corresponding octree box. The relative tolerance depicts the relative reduction of the residual at the approximate solution of the subdomain correction. We additionally vary the number of levels.

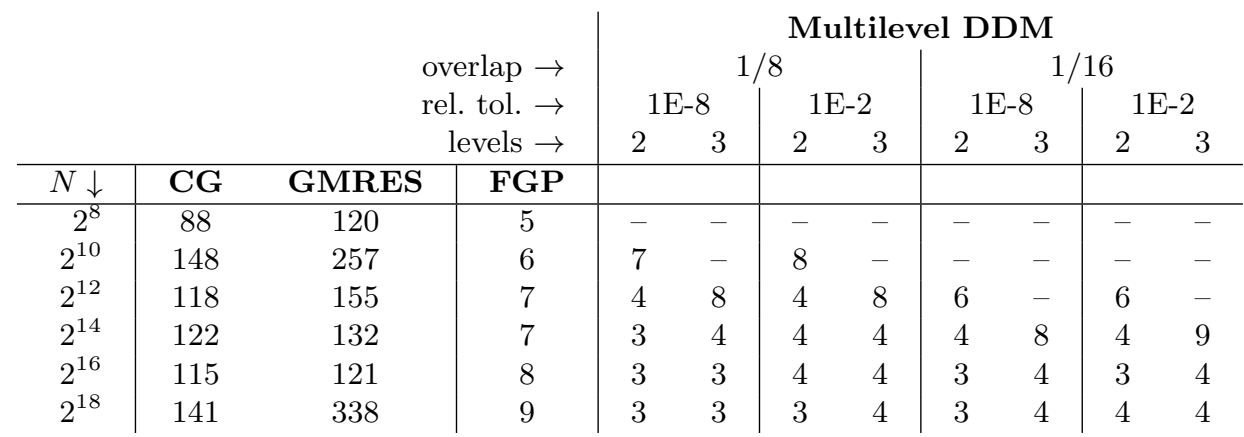

Table 1. Iteration count for various iterative methods.

The near constant iteration numbers indicate a sound preconditioning effect as soon as a sufficient overlap is created. However, the method fails to converge if no sufficient overlap is created.

We present timing results for two test systems in table 2 . The first test system is equipped with two IntelßXeonßE5-2640 v3 CPUs. We used an overlap of $1 / 8$ box size, a multilevel tree depth of $\max \left(1,\left\lceil\log _{8}(p)\right\rceil\right)$ for $p$ cores, and a relative tolerance of $10^{-2}$ for subsystems and an absolute tolerance of $10^{-6}$ for the total system. The column labeled 'sequential' lists the timings for the sequential code, the remaining columns state the timings for the MPI code running on the respective number of cores.

The second test system is a cluster of 16 Nvidia@Tegra $(\mathrm{X} 1$ SoC nodes, each equipped with 4-Plus-1 quad-core ARM Cortex A-57 CPUs [17]. We retained the settings as above except a multilevel tree depth of $\max \left(1,\left\lceil\log _{8}(p)\right\rceil\right)+1$ for $p$ cores. 


\begin{tabular}{|c|c|c|c|c|c|c|}
\hline \multicolumn{7}{|c|}{ Intel Xeon system } \\
\hline $\begin{array}{r}\text { cores } \rightarrow \\
N \downarrow\end{array}$ & sequential & 1 & 2 & 4 & 8 & 16 \\
\hline $2^{10}$ & $4.50 \mathrm{E}-2$ & $6.28 \mathrm{E}-2$ & $3.31 \mathrm{E}-2$ & $2.11 \mathrm{E}-2$ & $1.37 \mathrm{E}-2$ & $6.83 \mathrm{E}-3$ \\
\hline $2^{12}$ & $3.24 \mathrm{E}-1$ & $3.40 \mathrm{E}-1$ & $1.74 \mathrm{E}-1$ & $1.03 \mathrm{E}-1$ & $6.74 \mathrm{E}-2$ & $3.83 \mathrm{E}-2$ \\
\hline $2^{14}$ & $2.69 \mathrm{E}+0$ & $2.71 \mathrm{E}+0$ & $1.42 \mathrm{E}+0$ & $7.74 \mathrm{E}-1$ & $4.75 \mathrm{E}-1$ & $2.15 \mathrm{E}-1$ \\
\hline $2^{16}$ & $1.53 \mathrm{E}+1$ & $2.11 \mathrm{E}+1$ & $1.10 \mathrm{E}+1$ & $6.38 \mathrm{E}+0$ & $4.08 \mathrm{E}+0$ & $1.52 \mathrm{E}+0$ \\
\hline $2^{18}$ & - & $1.37 \mathrm{E}+2$ & $7.19 \mathrm{E}+1$ & $3.82 \mathrm{E}+1$ & $2.47 \mathrm{E}+1$ & $1.11 \mathrm{E}+1$ \\
\hline $2^{20}$ & - & - & - & $3.07 \mathrm{E}+2$ & $1.87 \mathrm{E}+2$ & $7.86 \mathrm{E}+1$ \\
\hline $2^{22}$ & - & - & - & - & - & $6.49 \mathrm{E}+2$ \\
\hline \multicolumn{7}{|c|}{ ARM system } \\
\hline $\begin{array}{r}\text { cores } \rightarrow \\
N \downarrow\end{array}$ & 4 & 8 & 16 & 24 & 32 & 48 \\
\hline $2^{14}$ & $2.64 \mathrm{E}+0$ & $1.82 \mathrm{E}+0$ & $1.66 \mathrm{E}+0$ & $1.52 \mathrm{E}+0$ & - & - \\
\hline $2^{16}$ & $1.79 \mathrm{E}+1$ & $1.14 \mathrm{E}+1$ & $7.38 \mathrm{E}+0$ & $6.79 \mathrm{E}+0$ & $8.17 \mathrm{E}+0$ & $7.58 \mathrm{E}+0$ \\
\hline $2^{18}$ & $1.37 \mathrm{E}+2$ & $7.18 \mathrm{E}+1$ & $3.49 \mathrm{E}+1$ & $3.25 \mathrm{E}+1$ & $3.50 \mathrm{E}+1$ & $3.20 \mathrm{E}+1$ \\
\hline $2^{20}$ & - & - & $1.59 \mathrm{E}+2$ & $1.30 \mathrm{E}+2$ & $1.17 \mathrm{E}+2$ & $9.66 \mathrm{E}+1$ \\
\hline
\end{tabular}

Table 2. Time per iteration on the two test systems.

The measured timings imply a quasilinear time complexity on both systems for the sequential and the distributed implementation. The method scales as long as an adequate workload remains per node.

\section{Conclusion}

We showed the applicability of our multilevel domain decomposition method using an octree as hierarchical structure. The near constant iteration numbers back up the reasonableness of the choice of the coarsening factor $1 / 8$ for this approach. Again, an approximate solution over the subdomains using the FGP algorithm proved useful. However, the area of application of our multilevel approach starts at $\approx 2^{10}$ boundary nodes. This clearly falls short of the threshold where distributed systems are demanded by our simulation applications.

We identified bottlenecks in the current communication design and the data division layout in our implementation that have to be addressed to further improve the scalability of our method. The optimization of the load balancing on heterogeneous systems utilizing different accelerators will also have an impact on the future data division layout.

\section{Acknowledgements}

This project has received funding from the European Unions Horizon 2020 research and innovation programme under grant agreement No 671697. 


\section{References}

1. Beatson, R.K., Greengard, L.: A short course on fast multipole methods. Wavelets, Multilevel Methods and Elliptic PDEs, pp. 1-37, Oxford University Press (1997).

2. Beatson, R.K., Light, W., Billings, S.: Fast Solution of the Radial Basis Function Interpolation Equations: Domain Decomposition Methods SIAM J. Sci. Comput., vol. 22,5 , pp. 1717-1740 (2001).

3. Beatson, R., Levesley, J., Mouat, C.: Better bases for radial basis function interpolation problems Comput. Appl. Math., vol. 236, pp. $434-446$ (2011).

4. de Boer, A., van der Schoot, M.S., Bijl, H.: Mesh Deformation Based on Radial Basis Function Interpolation. Comput. Struct., vol. 85, 11-14, pp. 784-795 (2007).

5. Bozzini, M.T., Rossini, M.F.: Multivariate approximation and interpolation with applications. Testing methods for 3D scattered data interpolation (Almunecar, 2001). Acad. Cienc. Exact.Fs.Qum. Nat., pp. 111-135, (2002).

6. Buhmann, M.: Radial Basis Functions. Theory and Implementations. Cambridge monographs on applied and computational mathematics, Cambridge University Press, New York (2003).

7. Cherrie, J.B., Beatson, R.K., Newsam, G.N.: Fast Evaluation of Radial Basis Functions: Methods for Generalized Multiquadrics in $\mathrm{Rn}$ SIAM J. Sci. Comput., vol. 23, 5, pp. 1549-1571 (2001).

8. Faul, A.C., Powell, M.J.D.: Krylov Subspace Methods for Radial Basis Function Interpolation. University of Cambridge, DAMP (1999).

9. Faul, A.C., Goodsell, G., Powell, M.J.D.: A Krylov subspace algorithm for multiquadric interpolation in many dimensions. IMA J. Numer. Anal., vol. 25, 1, pp. 1-24 (2005).

10. Fasshauer, G.: Meshfree approximation methods with MATLAB. World Scientific (2007).

11. Gumerov, N., Duraiswami, R.: Fast Radial Basis Function Interpolation via Preconditioned Krylov Iteration. SIAM J. Sci. Comput., vol. 29, 5, pp. 1876-1899 (2007).

12. Haase, G., Martin, D., Offner, G.: Towards RBF Interpolation on Heterogeneous HPC Systems LSSC'15, pp. 182-190 (2015).

13. Ling L., Kansa E.J.: Preconditioning for radial basis functions with domain decomposition methods. Math. Comput. Model., vol 40, 13, pp. 1413-1427 (2004).

14. Martin, D., Haase, G.: Interpolation with radial basis functions on GPGPUs using CUDA. Technical Report SFB-Report 2014-04, SFB MOBIS, University of Graz, (2014).

15. Powell, M.J.D.: Some algorithms for thin plate spline interpolation to functions of two variables. Adv. Comput. Math., pp. 303-319 (1993).

16. Smith, B.F., Bjørstad, P.E., Gropp, W.D.: Domain decomposition: parallel multilevel methods for elliptic partial differential equations. Cambridge University Press, New York (1996).

17. Rajovic, N., Paul M., Gelado I., Puzovic N., Ramirez A., Valero M.: Supercomputing with commodity CPUs: Are mobile SoCs ready for HPC?. Proceedings of the International Conference on High Performance Computing, Networking, Storage and Analysis (2013).

18. Wendland, H.: Scatterred Data Approximation. Cambridge monographs on applied and computational mathematics, Cambridge University Press, New York (2010). 